\title{
DNA regions that regulate the ovarian transcriptional specificity of Drosophila yolk protein genes
}

\author{
Susan K. Logan, ${ }^{1}$ Michael J. Garabedian, ${ }^{2,3}$ and Pieter C. Wensink ${ }^{2,4}$ \\ The Rosenstiel Center and the Departments of Biology ${ }^{1}$ and Biochemistry ${ }^{2}$, Brandeis University, Waltham, Massachusetts \\ 02254-9110 USA
}

Yolk protein genes 1 and 2 (yp1 and yp2) of Drosophila melanogaster are divergently transcribed neighboring genes. Both are transcribed in only two tissues, the ovarian follicle cells and the fat bodies of adult females. Previous work has identified a yolk protein enhancer between the genes that is sufficient to direct transcription in one of the tissues, female fat bodies. Using germ-line transformation methods, we identify two cis-acting regions with positive effects on transcription in ovaries. One, a 301-bp region located between the genes, influences both genes and is an enhancer determining the stage and cell type specificity of ovarian transcription. The other, a 105-bp region located in the first exon of yp2, acts across the yp2 promoter region to stimulate yp1 transcription in ovaries. Additional observations suggest how a single enhancer influences both promoters.

[Key Words: Tissue specificity; transcription; enhancer; Drosophila; yolk protein genes]

Received April 24, 1989; revised version accepted July 5, 1989.

Yolk protein genes 1 and 2 (yp1 and yp2) of Drosophila melanogaster code for two of the three abundant food proteins of the egg (Barnett et al. 1980). These two genes are regulated developmentally to have a highly specific pattern of transcription. They are transcribed only in adult females and only in two tissues, the fat bodies and the follicular epithelium of ovarian egg chambers (Barnett and Wensink 1981; Brennan et al. 1982; Garabedian et al. 1985, 1986). In addition, yolk protein transcription is limited to only part of the follicle cell lifetime. Follicle cells cease mitosis and cell division by stage 6 of oocyte development and continue to synthesize RNA and protein through stage $14, \sim 30 \mathrm{hr}$ later (King and Vanoucek 1960; King 1970; Margaritis et al. 1980; Griffin-Shea et al. 1982; Parks et al. 1986). The yolk protein transcripts first appear at stage 8 , are abundant from stages 9 through $10 \mathrm{~b}$, and are rare by stage 11 (Brennan et al. 1982). Thus, transcription of the two yolk protein genes is sex and tissue specific and has stage specificity within the lifetime of individual cells.

The developmental specificity of yolk protein gene expression is known to be influenced by several transacting components. In both follicle cells and fat bodies, yolk protein expression requires continuous activity of the tra 2 gene, one of the genes in the somatic sex-determination pathway (Belote et al. 1985). Other genes in this pathway also influence yolk protein expression (Postlethwait et al. 1980; Bownes and Nöthiger 1981). In

${ }^{3}$ Current address: Department of Biochemistry and Biophysics, University of California, San Francisco, California 94143 USA.

'Corresponding author. addition, the ecdysterone and juvenile hormones are required for normal levels of yolk protein transcripts (Postlethwait and Handler 1979; Jowett and Postlethwait 1980; Bownes et al. 1983). Although some of these trans-acting components may act indirectly, their influence on yolk protein genes demonstrates that multiple controls are needed for normal developmental expression and that at least one of these controls, tra2, is required continuously for expression.

Previous work has identified cis-acting regions necessary for the developmental specificity of yp1 and yp2 transcription (Garabedian et al. 1985, 1986; Shepherd et al. 1985). These two genes are transcribed divergently from cap sites that are 1224 bp apart (Fig. la; Hung and Wensink 1983). Germ-line transformation studies demonstrated that the DNA regions directing yolk protein transcription in each tissue are different. One region is a 125-bp enhancer that acts bidirectionally to direct transcription in adult female fat bodies; it is located between the two genes. The other region directs transcription in ovaries and is within a 2.8 -kb DNA fragment that includes yp2 and begins 886 bp upstream of yp 1 .

This paper describes the characterization of two ovarian regulatory regions located within the $2.8-\mathrm{kb}$ fragment. Characterization was accomplished by examining the in vivo expression pattern of deletion, substitution, and fusion gene constructs. The results demonstrate that both DNA regions are necessary for normal ovarian transcription. One region is between the two genes and acts as an enhancer to determine the stage and cell specificities of yolk protein ovarian expression. The results indicate that this enhancer normally has the 

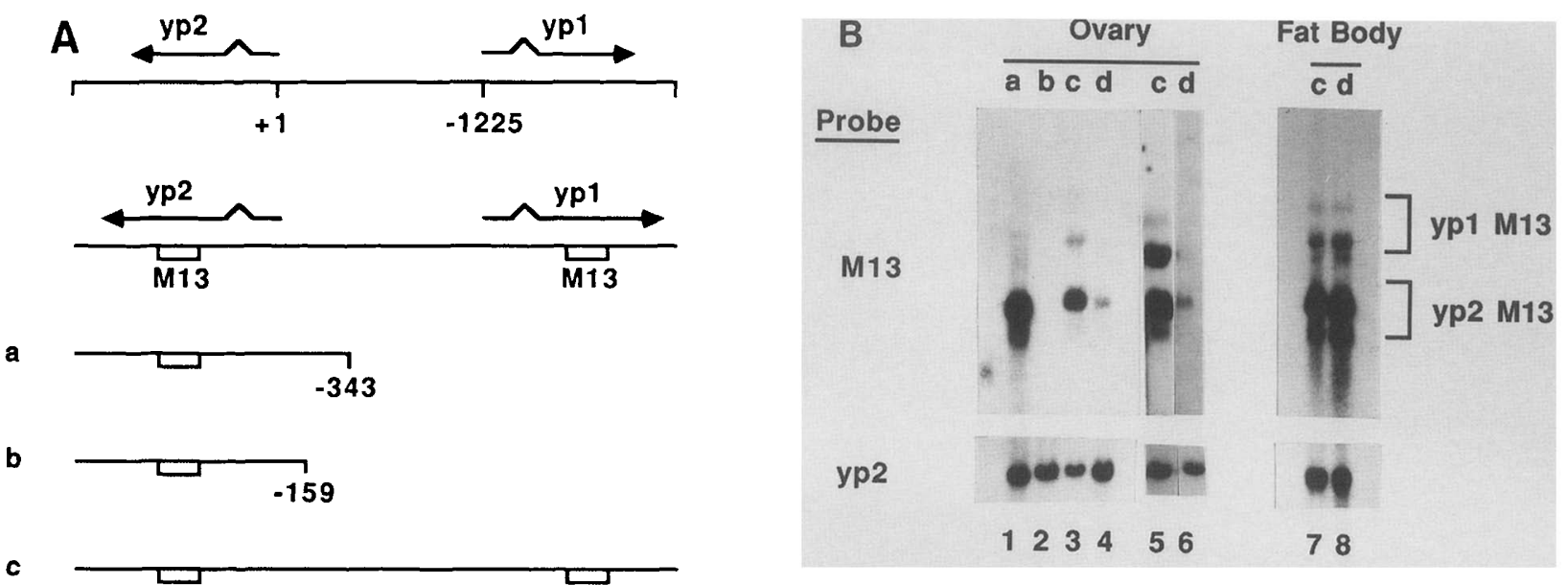

\section{d}

\section{$-159-340$}

Figure 1. Transcription patterns from deletions that demonstrate an upstream ovarian element. (A) The normal arrangement of ypl and yp2, the M13 insertions (open boxes), and the deletions whose end points are given in nucleotides relative to the yp2 cap site. (B) An autoradiogram of Northern blots of total RNA from transformed flies. The introduced yolk protein gene construct is indicated by the letter at the top of each lane. RNA is from dissected fat bodies $(2 \mu \mathrm{g}$ of RNA/lane) or ovaries $(5 \mu \mathrm{g}$ of RNA/lane). The M13 probe detects multiple transcripts from the introduced genes. The multiple transcripts result from transcription termination within the M13 fragment (Garabedian et al. 1985). Lanes 5 and 6 are darker exposures from an experiment similar to that shown in lanes 3 and 4. Below is an autoradiogram of the same blot with M13 radiolabel removed and rehybridized to a yp2 probe. This control indicates that there are similar amounts of total RNA in each lane.

same effect on both genes and no effect on fat body expression (diagramed in Fig. 5). The second DNA region is within the first exon of yp 2 and acts across the yp 2 promoter to increase the level of ypl transcription in ovaries. Other observations suggest how a single enhancer influences both promoters.

\section{Results}

A 184-bp region between yp1 and yp2 is necessary for high levels of ovarian-specific expression from both genes

Previous work localized sequences sufficient for high levels of ovarian-specific expression of ypl and yp2 to a fragment extending from $343 \mathrm{bp}$ upstream to $\sim 2500 \mathrm{bp}$ downstream of the yp2 cap site [Fig. 1A (structure a); Garabedian et al. 1985]. To localize the ovarian regulatory elements more precisely, we made deletions in this region and introduced the altered structures into the Drosophila germ line by the P-element transformation method (Spradling and Rubin 1982). In these experiments, the altered yolk protein genes were marked by inserting a bacteriophage M13 sequence that distinguishes the genes and their transcripts from endogenous counterparts. Southern blot analysis demonstrated that several independently transformed lines were established for each altered structure and that each line has a single, unrearranged copy of the structure (data not shown). For each deletion described below, multiple independently transformed lines were examined.

The region between -343 and -159 is necessary for high levels of ypl and yp2 transcription in ovaries /un- less otherwise noted, all locations are described relative to the yp2 cap site). When this region is deleted from the $5^{\prime}$ end of yp2, the level of yp2 transcripts is reduced at least 50-fold in ovaries (Fig. $1 \mathrm{~A}$ and B, lanes 1 and 2). To determine whether this effect is limited to ovarian tissue or to the yp2 gene, the same region was deleted from the ypl-yp2 gene pair in their normal arrangement. This deletion construct contains the previously characterized fat body enhancer and produces normal levels of yp 1 and yp2 RNAs in fat bodies, indicating that both promoters are functional and that the deleted region does not affect fat body expression (Fig. 1B, lanes 7 and 8). However, this deletion does cause substantial reduction in the ovarian levels of both ypl and yp2 transcripts (Fig. 1B, lanes 3-6). For each of these deletions, two independent lines were assayed and gave indistinguishable results. The reduction in yp2 transcript levels caused in this ypl-yp2 construct deletion is not as great as that observed in the yp 2 construct deletion (Fig. 1A and $B$, lanes $1-4)$. This difference indicates that either another ovarian element is located upstream of -343 or that the dissected ovarian tissue is contaminated by low levels of fat body tissue containing yolk protein transcripts. Whichever the case may be, we conclude that the 184 -bp region has a positive tissue-specific influence on the ovarian transcription of both genes and no apparent influence on the fat body transcription of either gene.

A 105-bp region within the first exon of yp2 is necessary for normal levels of yp1 expression in ovaries Previous work has shown that a fragment containing the 
entire ypl gene truncated at -340 expresses yp1 RNA in adult female fat bodies, but at very low levels or not at all in ovaries in all five of the independent lines [Fig. 2A (structure a) and B (ovary lanes 1-5); Garabedian et al. 1985,1986 ]. When additional DNA is placed upstream of this ypl fragment so that it extends to the yp2 cap site and contains the entire intergenic region, very low and apparently unchanged levels of ovarian transcripts are observed in all three of the independent lines (Fig. 2B, ovary lanes $6-81$. Thus, the region between the yp 2 cap site and -343 , a region shown in the previous section to be necessary for high levels of ypl and yp 2 transcription in ovaries, is not sufficient for high levels of ypl ovarian transcription. We conclude that DNA within or downstream of yp2 is also necessary for high levels of ovarian transcription.

A search for a regulatory element within the yp2 gene identified a sequence necessary for high and apparently normal levels of ypl expression in ovaries. This element is within the first $105 \mathrm{bp}$ of the first exon of yp2. When it is added to the ypl gene and intergenic region, the level of ypl transcripts in all three independent lines increases in ovaries, becoming equal to that of the complete yp $1-y p 2$ construct. The transcript level from one of these lines is shown in Figure 2B lovary lane 9 versus 10). Therefore, a region in the first exon of yp 2 is necessary for normal levels of ypl transcription in ovaries and must act across the intervening promoter region and intergenic DNA.

\section{The 105-bp region and the 340 -bp region immediately upstream of yp2 influence yp1 expression in fat bodies}

The 105-bp region within the first yp2 exon and the 340 bp region immediately upstream of yp 2 appear to have opposing effects on yp1 expression in fat bodies. As expected from previous results, the ypl gene truncated at position -340 produces a high level of ypl RNA in fat bodies. The level of ypl RNA is approximately equal to that produced by the entire yp1-yp2 gene pair (Fig. 2B, fat body lanes $1-5$ versus lane 10). However, when additional DNA is placed upstream of the ypl fragment so that it extends to the yp2 cap site, the amount of fat body RNA produced by each of the three independently transformed lines decreases substantially (Fig. 2B, fat body lanes $6-81$. When the ypl DNA is extended farther upstream to include the 105-bp region of yp2, ypl fat body RNA is restored to normal levels in all three independent lines. The transcript levels from one of these lines is shown in Figure $2 \mathrm{~B}$ (lane 9 versus lanes $1-5$ and 10). It might be concluded that an intergenic region has a negative effect on ypl expression in fat bodies and that this negative effect is overcome by the $105-\mathrm{bp}$ region that includes the cap site and part of the first exon of yp2. An alternative interpretation that bears on the mechanism by which a single enhancer acts on both promoters is offered in the Discussion.

The upstream ovarian region is an enhancer sufficient for the stage and cell specificity of yolk protein ovarian expression

Positive transcriptional effects of the region immediately upstream of yp 2 were assessed by testing its effect on a heterologous promoter from the Drosophila hsp 70 gene joined to a reporter gene, the $\beta$-galactosidase (lacZ) gene of Escherichia coli. This construct enables histochemical staining for $\beta$-galactosidase activity to be used as an assay for the stage- and tissue-specific effects of transcriptional regulatory elements (Garabedian et al. 1985). In the absence of heat shock, flies transformed with the $h s p-l a c Z$ gene produce only weak staining of the digestive system, a result also found in untransformed flies (Lis et al. 1983; Garabedian et al. 1986). If flies of the transformed line are heat shocked, almost all of the tissues of both sexes stain intensely, indicating that the fusion gene can produce functional $\beta$-galactosidase in almost all cell types, including ovarian follicle
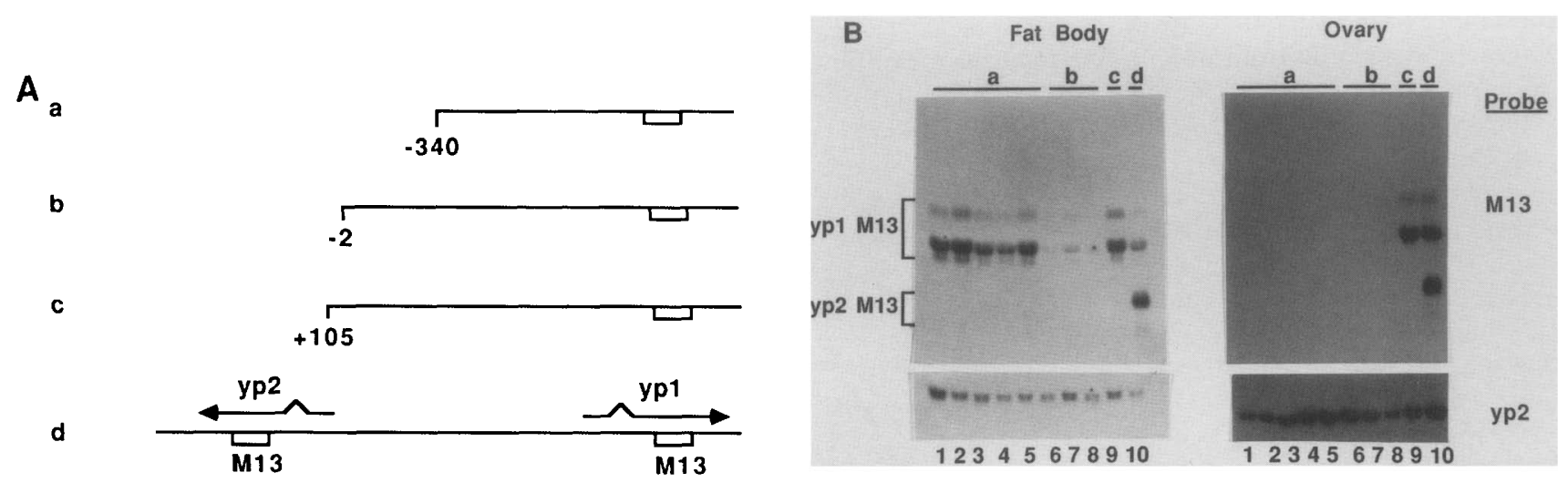

Figure 2. Transcription patterns from deletions that demonstrate a regulatory sequence within the yp2 gene. Labeling conventions are as in Fig. 1. $|A|$ The normal genomic arrangement of ypl and yp2 and the relevant deletions. $(B)$ An autoradiogram of Northern blots of total RNA from independently transformed flies. RNA is from dissected fat bodies $(2 \mu \mathrm{g}$ of RNA/lane) or ovaries $(5 \mu \mathrm{g}$ of RNA/lane). Gel blots were hybridized to radiolabeled M13 DNA. Below is an autoradiogram of the same blot with M13 radiolabel removed and rehybridized to a radiolabeled yp2 probe. This indicates that approximately twofold less RNA was loaded in lanes $4,6,8$, and 10 than in lanes 7 and 9. 
cells and adult fat bodies (Lis et al. 1983; Garabedian et al. 1986).

The yolk protein DNA fragment tested $(-343$ to -43$)$ contains the upstream region shown above to be necessary for ovary-specific expression of both ypl and yp2 and extends an additional $116 \mathrm{bp}$ to a position $13 \mathrm{bp}$ upstream of the yp2 TATA box (Hung and Wensink 1983). When this fragment is placed $196 \mathrm{bp}$ upstream of the $h s p-l a c Z$ gene and then transformed into the germ line, $\beta$-galactosidase activity occurs as a stripe, approximately one-third of the distance from the anterior tip of the ovaries in all three independent lines (Fig. 3A). Details of the ovarian staining pattern typical for this construct can be seen in Figure 3C, which shows an ovary from a fly transformed with a different fusion construct that gives the same staining pattern. Other than low levels of endogenous staining in intestines, staining does not occur elsewhere at any developmental stage and particularly not in adult female fat bodies where yolk protein genes normally are expressed. We conclude that this fragment from the intergenic region is sufficient to direct ovarian, but not fat body, expression from the heterologous $h s p 70$ promoter.

Examination of separated egg chambers reveals that the staining is similar or identical to the normal yolk protein expression pattern in all lines. Early egg chambers, stages $1-7$, show no $\beta$-galactosidase activity, whereas activity occurs over the oocyte portion of the chamber after stage 8 (Fig. 3D and E). This staining is limited to the follicle cells and is not detected in oocytes or nurse cells. At stage 10, staining over the central region of the oocyte begins to recede leaving behind a small patch of $\sim 30$ stained cells at the posterior tip (Fig. $3 \mathrm{D}$ ). By stage 13, the oocyte has expanded to occupy almost the entire egg chamber. At this time, the posterior tip and an anterior cap of $\sim 300$ follicle cells are still stained, whereas $\sim 500$ follicle cells covering the large central portion of the oocyte are unstained. The posterior tip, anterior cap, and central follicle cells appear to correspond to three subpopulations of follicle cells distinguished previously on morphological criteria (Mahowald and Kambysellis 1980; Margaritis et al. 1980). These subpopulations become morphologically distinct at approximately stage 11 .

We conclude that the 300-bp fragment (hereafter called the ovarian enhancer) is sufficient for the stage and cell specificity of yolk protein ovarian expression. In addition, the highly sensitive $\beta$-galactosidase assay appears to have revealed either subtleties of expression in follicle cell subpopulations that have not been observed previously or differential stability of the fusion protein or RNA in these different cell subpopulations.

The stage-specific effect of the ovarian enhancer on the hsp70 promoter is influenced by orientation

Other $h s p-l a c Z$ fusions were constructed and introduced into the germ line to investigate the orientation and position dependence of ovarian enhancer activity. The results described above suggest that the enhancer acts in both directions and at different distances from the two yolk protein promoters. However, when the region is inverted at a site $196 \mathrm{bp}$ upstream from the $h s p$ promoter, there appears to be an effect on its activity. In one orientation all three independently transformed lines express in the normal yolk protein ovarian pattern (Figs. $3 \mathrm{~A}$ and $\mathrm{E}$ and $4 \mathrm{a}$ ). Expression differs between the two fly lines with the ovarian enhancer region in the opposite orientation (Fig. 4b). One of these lines has the normal expression pattern, whereas the other stains weakly and only in the anterior follicle cells that migrate between the oocyte and the nurse cells. This suggestion of an orientation effect is strengthened by results obtained when the enhancer region is moved an additional $880 \mathrm{bp}$ upstream of the $h s p$ promoter. In these structures, the additional DNA separating the ovarian enhancer and $h s p-l a c Z$ structure is the yolk protein intergenic DNA normally found between the ovarian element and the ypl cap site (Fig. $4 \mathrm{c}$ and d). When the ovarian enhancer region is present in the same orientation and exactly the same location relative to the $h s p 70$ cap site as it normally is relative to the ypl cap site, normal ovarian expression occurs in two independent lines (Figs. 3B-D and 4c). When the region is inverted at this position, ovarian expression is still limited to follicle cells and is constant within a transformed line, but varies from line to line (Fig. $4 \mathrm{~d}$ ). The variability in the ovarian expression among five independent lines includes normal expression from egg chamber stages $8-10$, expression from egg chamber stages $8-14$, expression only in the anterior follicle cells, and expression only in late-stage egg chambers 13-14. Expression at stage 13 in a line that expresses in late-stage egg chambers is shown in Figure 3F. With the exception of Figure $3 \mathrm{~F}$, photographs are not shown because no single line is representative. This orientation effect appears specific to ovarian expression because all lines transformed with structures $c$ and d (Fig. 4) express at high levels in fat bodies as expected from the presence of the fat body enhancer and do not express in unexpected tissues.

We conclude that in one orientation and at different positions, the ovarian enhancer consistently directs a heterologous promoter to produce the yolk protein ovarian expression pattern. In the other orientation, expression is limited to the normal cell type but is expressed with different stage specificities or in only a subset of the follicle cells at different chromosomal sites. The altered specificities may be a result of increased sensitivity to regulatory elements at the different chromosomal sites of insertion.

\section{Discussion}

Two regions are necessary for yolk protein ovarian expression

The experiments described in this paper identify two cis-acting DNA regions necessary for efficient ovarian expression of Drosophila yolk protein genes. One of these is between the yp1 and yp2 genes and acts on both 
A
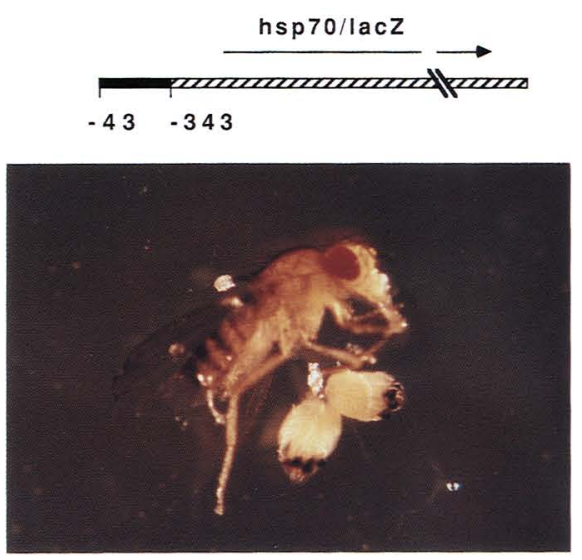

C

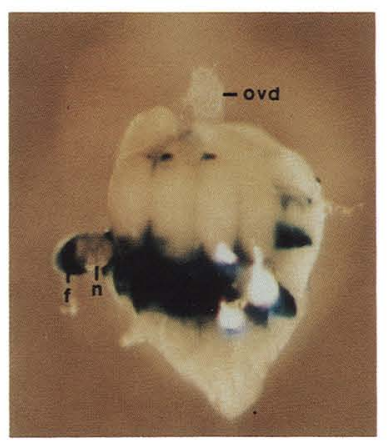

D

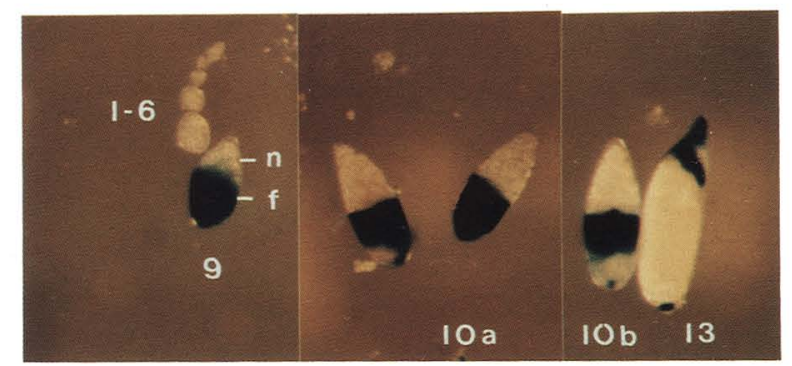

E

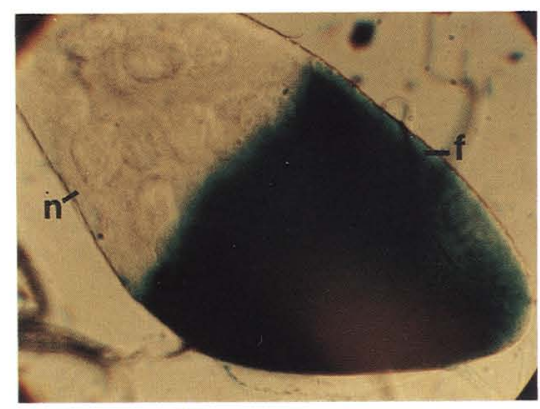

B
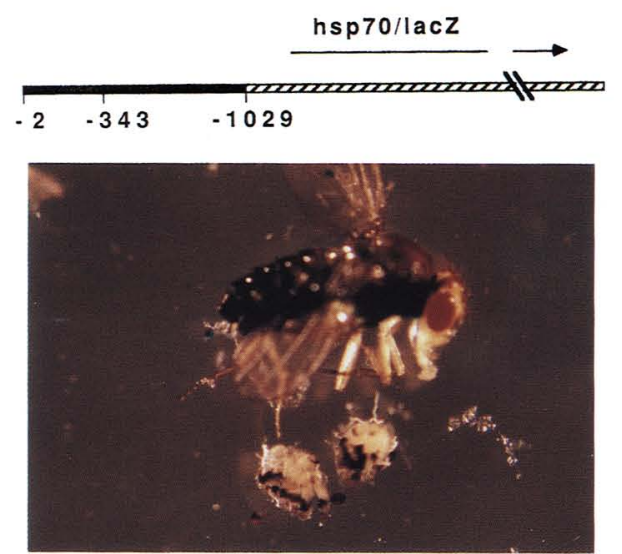

$10 \mathrm{a}$

$\mathbf{F}$

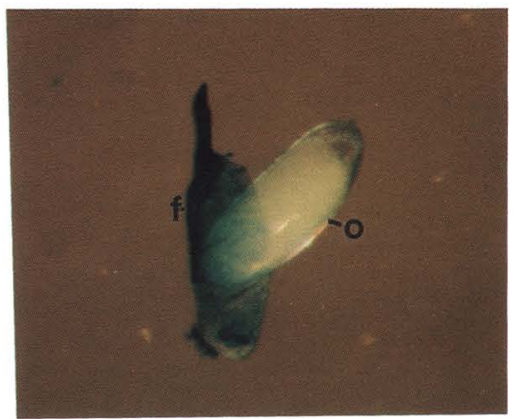

Figure 3. $\beta$-Galactosidase activity in flies transformed by yolk protein-hsp 70-lac $Z$ fusion genes. $(A$ and $B) \mathrm{X}$-Gal-stained female flies transformed with the construct shown above the photographs. Dissected ovaries are shown below each fly. $(C, D$, and $E)$ Ovarian staining patterns typical of flies transformed with DNA structures diagramed in $A$ and $B$. The photographs are from flies transformed with the structure diagramed in $A(E)$ and in $B(C$ and $D)$. (C) An ovary (anterior is at the bottom) with follicle cells (f), nurse cells (n), and oviduct (ovd) labeled. (D) Egg chambers (anterior at the top), with numbers indicating developmental stages according to King (1970). (E) An egg chamber viewed with Nomarski optics. (F) A stage-13 egg chamber (ol, with its follicle cell layer (f) pulled from the surface. This egg chamber is from a fly line transformed with the DNA structure diagramed in Fig. $4 \mathrm{~d}$. 


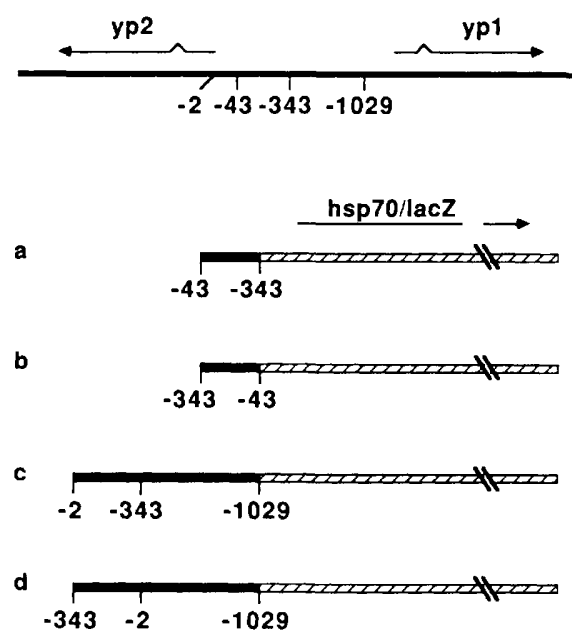

Figure 4. Constructs used to examine the position and orientation dependence of the ovarian enhancer. (Top) The normal genomic arrangement of yp1 and yp2. Below are constructs that fuse yolk protein DNA (heavy black line) to nucleotide - 195 of an $h s p 70-l a c Z$ fusion gene (hatched line).

genes. Our experiments demonstrate that an ovarian enhancer is sufficient to direct the yolk protein expression pattern in ovaries but not in fat bodies. The second region influencing ovarian expression is within the first yp2 exon and is called element I. Evidence presented in this paper demonstrates that element $I$ is necessary for high levels of ovarian transcription initiated from the yp1 promoter. Our recent unpublished results indicate that element I is an enhancer that can direct ovarian follicle cell specificity but not the normal yolk protein stage specificity of transcription.

\section{Stage and cell specificity of the ovarian enhancer}

Our experiments demonstrate that the ovarian enhancer activates transcription from the $h s p 70$ promoter in ovarian follicle cells. The pattern of transcription caused by this enhancer is similar to endogenous yolk protein transcription (Brennan et al. 1982). In both cases, expression begins at stage 8 and is strong from stages 9 to $10 \mathrm{~b}$ of egg chamber development. The only difference occurs after stage $10 \mathrm{~b}$. The dot blot and in situ hybridization experiments indicated that endogenous yolk protein transcripts are rare at stage 11 and absent thereafter (Brennan et al. 1982). In agreement with this, our assays of the fusion construct activity show expression halting over the major central portion $\{\sim 500$ cells $\}$ of the follicle cell layer. However, we also observe activity through stage 13 in subsets of follicle cells at the posterior $(\sim 30$ cells) and anterior ( $\sim 300$ cells). This unexpected expression in later stages might also occur with endogenous yolk protein genes but might only be detected with the fusion construct because of the high sensitivity of the $\beta$-galactosidase assay system. If this were the case, it would suggest that the two subpopulations of follicle cells, unlike the majority of follicle cells, do not repress yolk protein expression at later stages. Alternatively, the $h s p-\beta$-galactosidase mRNA or enzyme may have greater stability than yolk protein mRNA in these two cell subpopulations.

Although the ovarian enhancer causes a yolk protein pattern of expression from a heterologous promoter and does so at different positions, there is an orientation dependence to its influence (Fig. 4a and c). Apparently normal expression occurs when it is placed in the orientation it would normally have relative to ypl, but abnormal expression occurs when it is in a yp2 orientation (Fig. 4a-d). This orientation dependence was unexpected because deletions in the normal yp1-yp2 structure indicate that it acts bidirectionally on yp1 and yp2 promoters in ovaries (Fig. 1d). There are many possible explanations for the orientation-dependent influence of the ovarian enhancer region. One of the simplest is that the fragment used to reveal orientation dependence $(-343$ to -43 ; Fig. 4$)$ may contain remnants of the yp2 promoter region that interfere with enhancer activity.

Whatever the cause of the orientation dependence, we identified an enhancer that specifically influences expression in follicle cells and limits expression to only a portion of the lifetime of those cells. The regulatory system determining this follicle cell stage specificity may be related to that recently proposed to explain the timing of chorion gene expression in follicle cells (Mariani et al. 1988). The results reported here also indicate that the stage-specific regulation is different for different subpopulations of follicle cells.

We conclude that the ovarian enhancer is sufficient to direct a heterologous promoter to transcribe with the stage and cell specificity of yolk protein genes. Furthermore, because an entirely different mRNA is produced, yet mimics the yolk protein expression pattern, we conclude that on a qualitative level, both activation and repression of these genes in ovaries occurs at the level of transcription.

\section{A model for the influence of yolk protein regulatory elements}

Our current interpretation of the transcriptional influences of element I and the two cell type-specific enhancers is shown in Figure 5. The ovarian enhancer influences both promoters (Fig. 1). The fat body enhancer is likely to influence both, but influence has been demonstrated directly only for the ypl promoter. Its influence on the yp 2 promoter is likely because it acts bidirectionally and is the only intergenic sequence sufficient to direct fat body expression from a yolk protein or heterologous promoter (Garabedian et al. 1985, 1986; Shepherd et al. 1985). Element I influences the level of yp I transcripts in ovaries (Fig. 2). It may also influence the ovarian expression of yp2 because, as described above, our unpublished results demonstrate that it is a follicle cell enhancer.

We propose that the negative influence of the ovarian enhancer region on yp 1 fat body expression is an artifact of one of the deletion end points used. When a fragment containing the ovarian enhancer was placed in its 


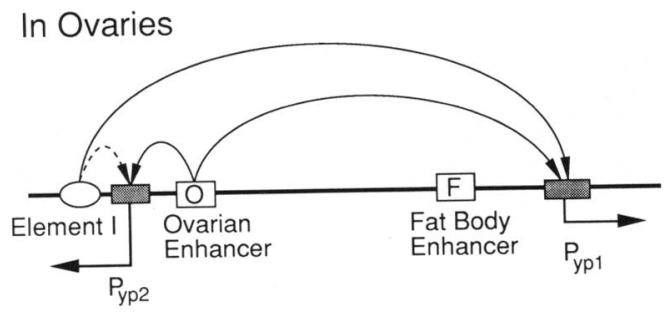

In Fat Bodies

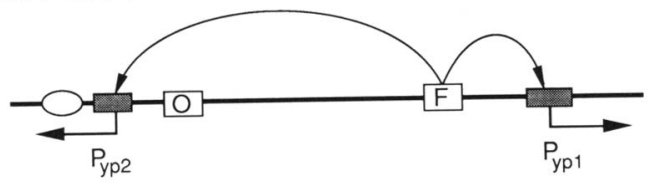

Figure 5. The influence of cis-acting DNA regions on yp1 and yp2 transcription. ypl-yp2 gene region (horizontal line); promoters (shaded boxes); cell type-specific enhancers (open boxes); element I (ovals). Curved solid arrows indicate positive influences on transcription. The dashed arrow indicates a hypothetical influence.

normal position relative to ypl, a decrease in ypl fat body expression occurred (Fig. 2). Addition of the element I region restored ypl fat body expression to normal level (Fig. 2). A simple hypothesis of alternative DNA loops can explain these results without requiring fat body specific activity of either the ovarian enhancer or element I. This hypothesis also suggests how a single enhancer could influence both promoters. We speculate that the normal gene structure and associated transacting factors maintain an equilibrium between alternative DNA loops in fat bodies (Fig. 6, top; Ho and Schleif 1988). In the normal gene structure, the ypl and yp2 promoters compete equally for interaction with the fat body enhancer, although only one interacts at any time. Figure 6 (bottom) shows how a deletion end point in the cap site may have caused the reduced expression in fat bodies observed in our deletion series (Fig. 2). The damaged promoter has an abnormal cap site without the downstream yp2 DNA, that is likely to contain promoter elements (Jones et al. 1988; Perkins et al. 1988; Soeller et al. 1988; Van Dyke et al. 1988|. We speculate that this damage stabilizes nonproductive interactions between the fat body enhancer and the yp 2 promoter, thereby shifting the normal equilibrium away from productive interactions with the yp 1 promoter, yielding the reduced transcript levels observed.

We speculate that the alternative loop model used to explain the interactions between the fat body enhancer and the yp1 and yp2 promoters also applies to the interaction between the ovarian enhancer and these two promoters. This speculation is based on the observation that damage to the ovarian enhancer has a strong negative effect on ovarian expression of both flanking yolk protein promoters (Fig. 1). A similar model has been proposed to explain transcriptional regulation of both $\beta$ and $\epsilon$-globin genes by the $\beta$-globin enhancer, although mutually exclusive loops rather than loops in equilibrium were necessary to explain the phenomena in the globin case (Choi and Engel 1988).

\section{Methods \\ DNA constructions}

The yolk protein DNAs used to make the ypl-Ml3 and yp2-M13 deletion constructs were derived from pYPl-M13 676 and pYP2-M13 ${ }_{676}$ (Garabedian et al. 1985). These deletion constructs were made by standard techniques (Schleif and Wensink 1981; Maniatis et al. 19821, using restriction sites found in the complete sequence of this region (Hung and Wensink 1981, 1983). The yolk protein regions then were cloned into the transformation vector CP20.1 (Simon et al. 1985). Restriction enzymes used in these constructions were purchased from New England Biolabs and Boehringer-Mannheim. Digestion conditions were those recommended by commercial suppliers.

The yolk protein $h s p-l a c Z$ fusion genes were made by placing yolk protein DNA fragments at a site - 196 upstream of a hybrid gene that fuses the upstream region and the $5^{\prime}$ end of the Drosophila hsp70 gene to the protein-coding portion of the E. coli lacZ gene (Lis et al. 1983). The constructions were made essentially as described by Garabedian et al. (1986). Unless otherwise noted, yolk protein fragments were cloned using known restriction sites. The deletion at -43 upstream of yp2 was made by Bal31 enzyme digestion, followed by the addition of a
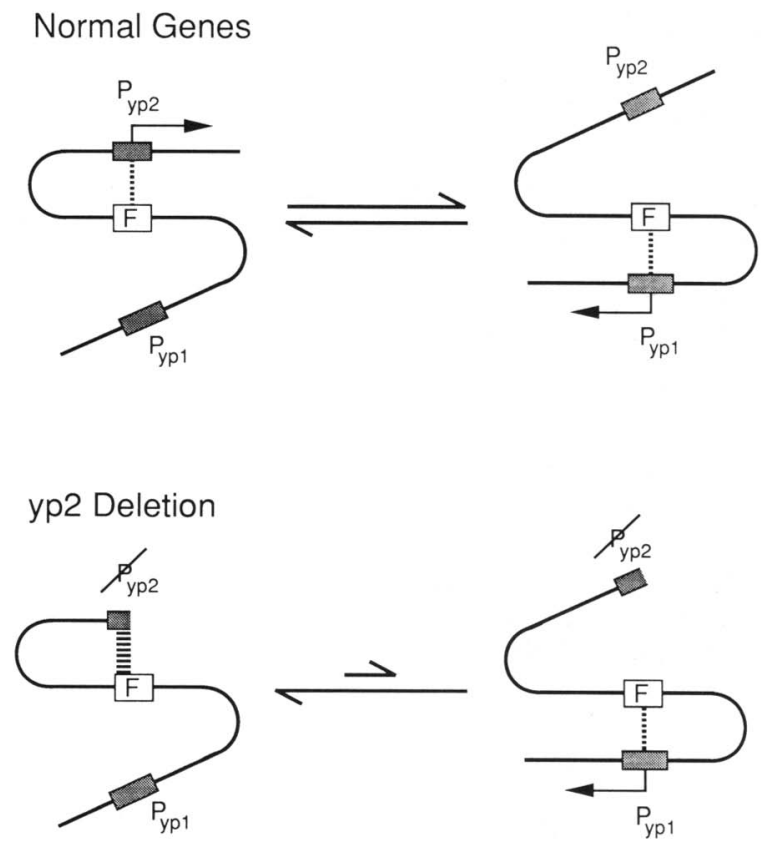

Figure 6. Model for interaction between the fat body enhancer and the two yolk protein gene promoters. Equilibria between alternative DNA loops are shown for the normal genes and for a deletion that damages the yp 2 promoter (F). The solid line represents the ypl and yp 2 genes, and the dashed lines represent protein-mediated interactions that are hypothesized the occur in fat bodies between the body enhancer and the yolk protein gene promoters. The thickness of the dashed lines indicates the strength of the interaction. In this model only promoters with an angular arrow are transcribed. Enhancer and promoters are symbolized as in Fig. 5. 
SalI linker to the resected end. This DNA was sequenced to determine the exact location of the breakpoint and the introduced Sall site.

\section{Germ-line transformation and analysis of transformants}

Transformation procedures were carried out according to Spradling and Rubin (1982). Embryos from a ry ${ }^{-}$strain of Drosophila (ry $\Delta^{506}$ ) were injected with the ypl-yp2 constructs at 350 $\mu \mathrm{g} / \mathrm{ml}$ and a defective P-element helper $\mathrm{p} \pi 25.7$ w.c. (Karess and Rubin 1984) at $150 \mu \mathrm{g} / \mathrm{ml}$. Total RNA was prepared (Barnett et al. 1980) from tissues dissected from 3- to 5-day-old adult flies. The techniques used for isolating and examining nucleic acids have been described by Kalfayan and Wensink (1982) and Garabedian et al. (1985). All Northern and Southern blots were hybridized to ${ }^{32} \mathrm{P}$-labeled DNAs (probes). The X-Gal assay for $\beta$ galactosidase activity was performed as described by Lis et al. (1983).

\section{Acknowledgments}

We thank Bob Schleif, Mike Wormington, Susan Hardin, Karen Coschigano, Kim O'Donnell, Doug Jacoby, Marie Lossky, and David Epstein for critical comments on the manuscript. We are grateful to Karen Coschigano for help in sequence determination and to Marty Stanton for constructing and transforming the yp2-M13 - 159 deletion. This work was supported by a grant from the National Institutes of Health (R01-GM21626).

\section{References}

Barnett, T. and P.C. Wensink. 1981. Transcription and translation of yolk protein mRNA in the fat bodies of Drosophila. In Developmental biology using purified genes. UCLA-ICN Symposium (ed. D.D. Brown and C.F. Fox), vol. 23, pp. 97-106. University of California Press, Berkeley, California.

Barnett, T., C. Pachl, P.J. Gergen, and P.C. Wensink. 1980. The isolation and characterization of Drosophila yolk protein genes. Cell 21: 729-738.

Belote, J.M., A.M. Handler, M.F. Wolfner, K.J. Livak, and B.S. Baker. 1985. Sex-specific regulation of yolk protein gene expression in Drosophila. Cell 40: 339-348.

Bownes, M. and R. Nöthiger. 1981. Sex-determining genes and vitellogenin synthesis in Drosophila melanogaster. Mol. Gen. Genet. 182: 222-228.

Bownes, M., M. Blair, R. Kozma, and M. Dempster. 1983. 20hydroxyecdysone stimulates tissue specific yolk protein gene transcription in both male and female Drosophila. $J$. Embryol. Exp. Morphol. 78: 249-268.

Brennan, M.D., A.J. Weiner, T.J. Goralski, and A.P. Mahowald. 1982. The follicle cells are a major site of vitellogenin synthesis in Drosophila melanogaster. Dev. Biol. 89: 225-236.

Choi, O.-R.B. and J.D. Engel. 1988. Developmental regulation of $\beta$-globin gene switching. Cell 55: 17-26.

Garabedian, M.J., M.C. Hung, and P.C. Wensink. 1985. Independent control elements that determine yolk protein gene expression in alternative Drosophila tissues. Proc. Natl. Acad. Sci. 82: 1396-1400.

Garabedian, M.J., B.M. Shepherd, and P.C. Wensink. 1986. A tissue-specific transcriptional enhancer from the Drosophila yolk protein I gene. Cell 45: 859-867.

Griffin-Shea, R., G. Thireos, and F.C. Kafatos. 1982. Organization of a cluster of four chorion genes in Drosophila and its relationship to developmental expression and amplification. Dev. Biol. 91: 325-336.

Ho, L. and R.F. Schleif. 1988. Alternative DNA loops regulate the arabinose operon in Escherichia coli. Proc. Natl. Acad. Sci. 85: 5444-5448.

Hung, M.-C. and P.C. Wensink. 1981. The sequence of the Drosophila melanogaster gene for yolk protein 1. Nucleic Acids Res. 9: 6407-6419.

- 1983. Sequence and structure conservation in yolk proteins and their genes. I. Mol. Biol. 164:481-492.

Jones, K.A., P.A. Luciw, and N. Duchange. 1988. Structural arrangements of transcription control domains within the $5^{\prime}$ untranslated leader regions of the HIV-1 and HIV-2 promoters. Genes Dev. 2: 1101-1114.

Jowett, T. and J.H. Postlethwait. 1980. The regulation of yolk protein synthesis in Drosophila ovaries and fat body by $20-$ hydroxyecdysone and juvenile hormone analog. Dev. Biol. 80: 225-234.

Kalfayan, L. and P.C. Wensink. 1982. Developmental regulation of Drosophila $\alpha$-tubulin genes. Cell 29: 91-98.

Karess, R.E. and G.M. Rubin. 1984. Analysis of P transposable element functions in Drosophila. Cell 38: 135-146.

King, R.C. 1970. Ovarian development in Drosophila melanogaster. Academic Press, New York.

King, R.C. and E.G. Vanoucek. 1960. Oogenesis in adult Drosophila melanogaster. X. Studies on the behavior of the follicle cells. Growth 24: 333-338.

Lis, J.T., J.A. Simon, and C.A. Sutton. 1983. New heat shock puffs and $\beta$-galactosidase activity resulting from transformation of Drosophila with an hsp70-lacZ hybrid gene. Cell 35: 403-410.

Mahowald, A.P. and M.P. Kambysellis. 1980. Oogenesis. In The genetics and biology of Drosophila (ed. M. Ashburner and T. Wright), vol. 2d, pp. 141-224. Academic Press, New York.

Maniatis, T., E.F. Fritsch, and J. Sambrook. 1982. Molecular cloning: A laboratory manual. Cold Spring Harbor Laboratory, Cold Spring Harbor, New York.

Margaritis, L.H., F.C. Kafatos, and W.H. Petri. 1980. The eggshell of Drosophila melanogaster I. Fine structure of the layers and regions of the wild type eggshell. I. Cell Sci. 43: $1-35$.

Mariani, B.D., I.R. Lingappa, and F.C. Kafatos. 1988. Temporal regulation in development: Negative and positive cis regulators dictate the precise timing of expression of a Drosophila chorion gene. Proc. Natl. Acad. Sci. 85: 3029-3033.

Parks, S., B. Wakimoto, and A.C. Spradling. 1986. Replication and expression of an X-linked cluster of Drosophila chorion genes. Dev. Biol. 117: 294-305.

Perkins, K.K., G.M. Dailey, and R. Tjian. 1988. In vitro analysis of the Antennapedia P2 promoter: Identification of a new Drosophila transcription factor. Genes Dev. 2: 1615-1626.

Postlethwait, J.H. and A.M. Handler. 1979. The roles of juvenile hormone and 20-hydroxyecdysone during vitellogenesis in isolated abdomens of Drosophila melanogaster. I. Insect Physiol. 25: 455-460.

Postlethwait, J.H., M. Bownes, and T. Jowett. 1980. Sexual phenotype and vitellogenins in Drosophila. Dev. Biol. 79: 379387.

Schleif, R.F. and P.C. Wensink. 1981. Practical methods in molecular biology. Springer-Verlag, New York.

Shepherd, B.S., M.J. Garabedian, M.-C. Hung, and P.C. Wensink. 1985. Developmental control of Drosophila yolk protein 1 gene by cis-acting DNA elements. Cold Spring Harbor Symp. Quant. Biol. 50: 521-526.

Simon, J.A., C.A. Sutton, R.B. Lobell, R.L. Glaser, and J.T. Lis. 1985. Determinants of heat shock induced chromosome puffing. Cell 40: 805-817.

Soeller, W.C., S.J. Poole, and T. Kornberg. 1988. In situ transcription of the Drosophila engrailed gene. Genes Dev. 2: $68-81$. 
Spradling, A. and G.M. Rubin. 1982. Transposition of cloned P elements into Drosophila germline chromosomes. Science 218: $341-347$.

Van Dyke, M.W., R.G. Roeder, and M. Sawadogo. 1988. Physical analysis of transcription preinitiation complex assembly on a class II gene promoter. Science 241: 13351338. 


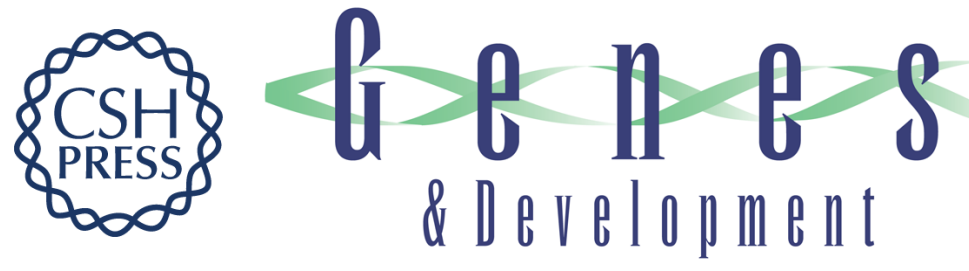

\section{DNA regions that regulate the ovarian transcriptional specificity of Drosophila yolk protein genes.}

S K Logan, M J Garabedian and P C Wensink

Genes Dev. 1989, 3:

Access the most recent version at doi:10.1101/gad.3.9.1453

References This article cites 29 articles, 10 of which can be accessed free at: http://genesdev.cshlp.org/content/3/9/1453.full.html\#ref-list-1

License

Email Alerting

Service

Receive free email alerts when new articles cite this article - sign up in the box at the top right corner of the article or click here.

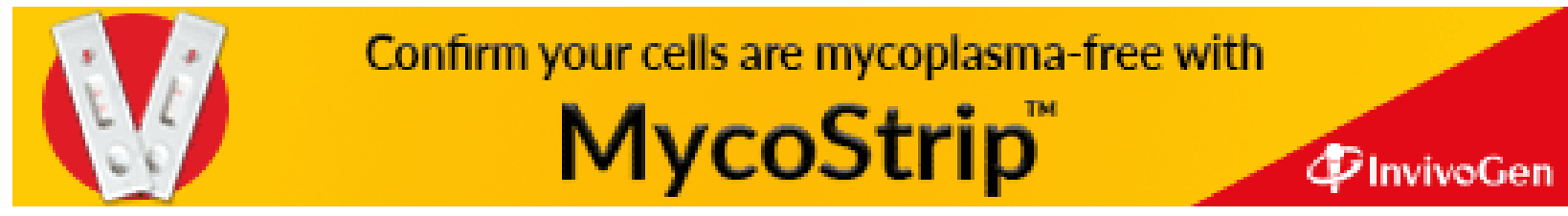

